\title{
Kinetic electronics: Monolithic processing of a layered flexible robotic actuator film for simple film microrobot fabrication
}

Shiyi Zhang

Kyushu University https://orcid.org/0000-0002-8466-3359

Joseph Wang

University of California, San Diego

Kenshi Hayashi

Kyushu University

Fumihiro Sassa ( $\nabla$ sassa@ed.kyushu-u.ac.jp )

Kyushu University https://orcid.org/0000-0002-3910-2931

\section{Article}

Keywords: Soft Robotic Techniques, Body-machine Interfaces, Actuation Durability, Integrated Electrical Circuits, Wearable Sensing Devices

Posted Date: May 28th, 2021

DOl: https://doi.org/10.21203/rs.3.rs-546958/v1

License: (9) This work is licensed under a Creative Commons Attribution 4.0 International License. Read Full License

Version of Record: A version of this preprint was published at Scientific Reports on October 8th, 2021. See the published version at https://doi.org/10.1038/s41598-021-99500-9. 
1 Title: Kinetic electronics: Monolithic processing of a layered flexible robotic actuator film for 2 simple film microrobot fabrication

3 Authors: Shiyi Zhang ${ }^{1}$, Joseph Wang ${ }^{2 *}$, Kenshi Hayashi ${ }^{1}$ and Fumihiro Sassa ${ }^{1 *}$

4 Affiliations:

$5{ }^{1}$ Graduate School of Information Science and Electrical Engineering, Kyushu University, 744

6 Motooka, Nishi-ku, Fukuoka, 819-0395 Japan,

$7 \quad 2$ Department of Nanoengineering, Center of Wearable Sensors, University of California San

8 Diego, La Jolla, CA, USA

$9 \quad *$ Corresponding authors

Abstract

Low-invasive soft robotic techniques can potentially be used for developing next-generation body-machine interfaces. Most soft robots require complicated fabrication processes involving 3D printing and bonding/assembling. In this letter, we describe a monolithic soft microrobot fabrication process for the mass production of soft film robots with a complex structure by simple 2D processing of a robotic actuator film. The $45 \mu \mathrm{g} / \mathrm{mm}^{2}$ lightweight film robot can be driven at a voltage of CMOS compatible $5 \mathrm{~V}$ with $0.15 \mathrm{~mm}-1$ large curvature changes; it can generate a force 5.7 times greater than its self-weight. In a durability test, actuation could be carried out over 8000 times without degradation. To further demonstrate this technique, three types of film robots with multiple degrees of freedom and moving illuminator robot were fabricated. This technique can easily integrate various electrical circuits developed in the past to robotic systems and can be used for developing advanced wearable sensing devices; It can be called "Kinetic electronics."

\section{Text}

\section{Introduction}

Advanced assistive devices such as non-invasive sensors, intelligent eye glasses, and robot suits support disabled and elderly people and expansion of all human activity ${ }^{1,2,3}$. For example, wearable chemical or biological sensors based on flexible electronics ${ }^{4,5,6,7}$ can monitor human health and activity and obtain environmental information about a person's surroundings ${ }^{8,9,10,11}$. A major bottleneck in the application of such devices to humans is the body-machine interface. Mismatches exist between the living body, which is always deforming or growing, and the unmoving rigid machines. The use of soft robots ${ }^{12,13}$ with movement similar to that of living organisms that have a flexible body is a promising approach for solving this problem. Soft robots consist of a soft material body and flexible actuators such as electroactive polymers (EAPs) ${ }^{14,15}$, pneumatic actuators ${ }^{16,17,18}$, electrothermal actuators ${ }^{19,20,21}$, or hydrogel ${ }^{22,23}$. They are resistant to mechanical impact and deformation and enable soft creature-like movement ${ }^{24}$. Thus, the use 
of soft robot techniques is a promising way to develop body-friendly creature-like body-machine

2 interfaces, which can allow sensing and movement and adjust a body's motion with physically 3 and chemically sensing ${ }^{25}$. Most current soft robot systems require complicated assembly 4 processes or special 3D fabrication processes ${ }^{12}, 26,27$, which incurs a high cost. Here, we demonstrate a low-cost monolithic soft microrobot fabrication process for the mass production of soft film robots with complicated structures by simple 2D processing of a robotic actuator film. The concept of a monolithically processed soft film robot is shown in Fig. 1(a). The film robot consisted of layers with different functions, including a flexible electric actuator layer and an extra functional layer. The actuator layer drives the motion of the film and other mechanical motion. The extra functional layer can be used for the heater, sensor, illumination, microflow channel, bone structure, etc. The film was temporally applied to support the substrate and then processed. In this study, a bimorph flexible film was employed as the actuator layer. The film can be used as an electrothermal actuator, an electrical film substrate, and a robot body when an electric circuit layer is formed on the film.

\section{Results and Discussion}

Processing of actuator film for developing a microrobot. The monolithic fabrication process of a film microrobot is shown in Fig. 1(b). First, the actuator film prepared by adhesive bonding $(($ Fig. 1(b(i-ii)))) was applied to a cutting sheet, which worked as a flexible carrier substrate with temporary adhesive. Then, the surface of the actuator film was wiped with $99.9 \%$ ethanol. The film fixed on the cutting sheet was set to a cutting plotter (STiKA SV-8, Roland, Japan) and cut according to the designed robot outline pattern (Fig. 1(b(iii))). Next, a stenciled mask for the heater electrode pattern was prepared with the same cutting plotter with an adhesive cutting sheet. The stenciled mask was aligned manually and applied to the surface of the actuator film (Fig. 1(b(ivv))). Then, a Au electrical layer $(50 \mathrm{~nm}$ ) was deposited to the sheet by a sputtering machine (SC$701 \mathrm{HMClI}$, Sanyu electronics, Japan) (Fig. 1(b(vi))). The stenciled mask was removed, and the film robot was taken off from the carrier substrate sheet (Fig. 1(b(vii))). Then, poly(dimethylsiloxane) (10\%; PDMS; KE-1300T, Shin-Etsu Chemical, Japan) diluted with hexane (085-00416, Fujifilm Wako Pure Chemical Corporation, Japan) was coated by using the dip coating method (Fig. 1(b(viii)). The surface was dried and cured at room temperature overnight to form a thin PDMS layer. The PDMS layer was formed to prevent mechanical damage the Au layer; it also worked as a mechanical buffer layer to other stacked layers owing to its low Young's modulus. Finally, the contact pad of the heater electrode was connected to an electrical wire by a conductive adhesive (DOTITE D-362, Fujikura kasei, Japan) to connect the driving circuit. Figure 1 (c) shows the design and photograph of a single-joint single-finger film microrobot formed by this process. 
2 Evaluation of thermal deformation of bimorph actuator films. To evaluated thermal 3 deformation of bimorph actuator films. The five types of bimorph actuator films prepared exhibited 4 bending deformation to a constant curvature at a constant corresponding temperature. They returned to their original curvature at room temperature after cooling down. The curvature $1 / \mathrm{r}$ of a strip-shaped thin bimorph actuator can be calculated considering the mechanics of material modeling ${ }^{28,29}$. It can be expressed as a function of temperature through the following equation.

$$
\frac{1}{r}=\frac{6 E_{1} E_{2} t_{1} t_{2}\left(t_{1}+t_{2}\right)\left(\alpha_{1}-\alpha_{2}\right) \Delta T}{\left(E_{1} t_{1}^{2}\right)^{2}+\left(E_{2} t_{2}^{2}\right)^{2}+2 E_{1} E_{2} t_{1} t_{2}\left(2 t_{1}^{2}+3 t_{1} t_{2}+2 t_{2}^{2}\right)}
$$

Here, $\Delta T$ is the temperature difference from original (bonding) temperature, $\alpha$ is coefficient of thermal expansion, $t$ is thickness of the film, and $E$ is the Young's modulus.

Photographs of the deformation of the films are presented in Fig. 2(a). Figure 2(b) shows the theoretical and experimental changes in the curvature of each film at different temperatures. The initial curvature of each specimen at room temperature $\left(25^{\circ} \mathrm{C}\right)$ was approximately $0.01 \mathrm{~mm}^{-1}$; however, the curvature at the maximum temperature $\left(100^{\circ} \mathrm{C}\right.$ in this experiment) varied greatly from 0.01 to $0.2 \mathrm{~mm}^{-1}$. PI/OPP, AL/PI, and Paper/OPP films showed a linear change in their curvature with the temperature range in this experiment, and the curvature values of PI/OPP and AL/PI were in good agreement with the theoretical values. In contrast, the rate of curvature change of $\mathrm{PI} / \mathrm{Paper}$ and $\mathrm{Al} / \mathrm{OPP}$ decreased from approximately $50{ }^{\circ} \mathrm{C}$. This may be due to the instable adhesion between the base films. The high-performance PI/OPP bimorph actuator film was used as the actuation layer in the film microrobot experiments.

Characteristics of robotic film actuation. To evaluate the basic actuation characteristics of the film microrobots, a single-finger robot shown in Fig. 1(c) was used. For each test, one new robot film was used, and the experiment was repeated five times.

Relationship between deformation and applied power. To determine the relationship between the film microrobot deformation and applied power, a constant power was applied to the robot film, and the curvature was measured when the film reached a steady state of heat dissipation and heat supply following the start of thermal deformation and stopped when a constant deformation was observed. The drive voltage was fixed at $5 \mathrm{~V}$ and the power supply to the heater was controlled by pulse width modulation (PWM; Fig. 3(a)). The changes in curvature are shown in Fig. 3(b). The curvature of the film robot showed a linear relationship with the power supplied with good reproducibility (worst case: relative STD $4.7 \%$ at $100 \%$ duty ratio). In the same way, 
1 the curvature of the robot was measured using a DC drive in the range of 5-6.6 V (Fig. 3(b)inset);

2 the curvature also showed a linear relationship with the supplied power.

3

4 Evaluation of speed of film robot actuation. To evaluate the robot speed, the frequency response of the robot deformation by a DC pulse signal was measured. A $5 \vee D C$ pulse with a pulse width of $25 \%$ of the cycle time was applied and the change in curvature and the movement of the robot tip were measured in the range of $0.125-10 \mathrm{~Hz}$. Figure 3(c) and Movie S1 shows the maximum and minimum values of the robot curvature in each cycle. The deflection of robot tip $d$ is also shown. At the lowest frequency in this experiment $(0.125 \mathrm{~Hz})$, the difference in the robot curvature between the maximum and minimum values was approximately $0.045 \mathrm{~mm}^{-1}$. However, the amount of change rapidly decreased as the frequency increased. Considering that the curvature of the electrothermally driven bimorph is a function of temperature change, it is believed that the shorter the heat-dissipation cycle, the smaller the change in temperature. In general, electrothermally driven bimorphs can be heated rapidly using a high voltage; however, the rate of heat dissipation is determined by the difference in temperature from the atmosphere, which limits their operating speed ${ }^{19}$.

Relationship between generated force and applied power. The force generated when the displacement of the robot tip was zero was measured. Figure 3(d) shows the force generated by the robot when driven with a $5 \vee$ PWM power supply at duty ratios of $0 \%-100 \%$. The force generated by the robot increased monotonically with increasing supplied power. The maximum force generated was $78 \mathrm{mgf}$, which is 5.7 times greater than the weight of the robot film (14 mg). In a low power range, no visible shape deformation of the robot was observed. However, with an increase in the supplied power, the tip of the film in the contact area between the robot and the plastic post was deformed, and the contact area increased. This deformation is thought to be the cause of the decreasing rate of generated force with supplied power.

Repetitive actuation durability of robot. To evaluate the durability of the robot, high-count repetitive bending actuation was performed. Here, a $5 \mathrm{~V} \mathrm{DC}$ pulse with a pulse width of $25 \%$ of the cycle time was applied, and 20000 repetitive motions were recorded with a video camera. The five-pulse average of the deflection of the robot tip is shown in Fig. 3(e). During the initial 2000 repetitive motions, the amplitude decreased monotonically. Then, it stabilized at a constant value until 10000 repetitions. After that, the amplitudes rapidly decreased. At this time, the initial heater resistance was $150 \Omega$; the final resistance after 20000 repetitions was $149 \Omega$, which is nearly the same as the initial resistance. The decrease in the amplitude may be owing to the aging process during the initial stage, such as microscale adhesion damage to the robot film. The results 
1 indicated that this simple film could be used for precise bending operation for approximately 8000

2 cycles with appropriate aging treatment.

3

4 Demonstration of film robots with multiple degree-of-freedom actuation. To demonstrate the proposed film robot fabrication process, we fabricated multi-degree-of-freedom robots with various electrode patterns and shapes. Robots can be fabricated by simply changing the design patterns in the process shown in Fig. 1(b). The design and operation photographs of the robots fabricated in this study are shown in Fig. 4. The two-joint single-fingered robot (Fig. 4(a), Movie S2) with a series of heater electrodes can move the robot tip to a point on a plane by heating two heaters independently. The finger array, which has four independent fingers (Fig. 4(b), Movie S3), can be driven up and down to perform contact operations on flat objects. It can be applied to large-scale multiplexing of cell colony pickers and reagent addition probes used in biological experiments $^{30}$. A gripper with a pair of opposing fingers (Fig. 4(c), Movie S4) can grasp and release an object precisely by manipulating the left and right fingers independently. It was used to grasp a styrofoam cube weighing $6 \mathrm{mg}$. The gripper was fixed to the tip of an optical microscope probe, so that the robot could be operated while observing the object, as shown in the microscope image in Fig. 4(c)(iii-v (inset)). A mobile illuminator film robot was also fabricated (Fig. 4(d), Movie S5). A blue color surface-mounted chip LED (SMLM12ABC7W1, Rohm semiconductor, Japan) with the dimensions of $2 \times 1.25 \mathrm{~mm}^{2}$ was manually mounted on the robot film using a conductive adhesive. The robot illuminated an object from different positions during robot motion ((Fig. 4(c(iiv)))). The blinking pattern and light intensity were controlled independently of robot motion. Such a robot can be used for the examination of part failure inside device housing using the circuit or for cavitas detection ${ }^{31,32}$ in human body by wearable moving sensors.

\section{Conclusion}

We developed a monolithic soft microrobot fabrication process for the mass production of lowcost robots with a complicated structure by simple $2 \mathrm{D}$ processing of a robotic actuator film. We investigated several materials for the base bimorph actuator film and evaluated and demonstrated the characteristics of the PI/OPP film, which showed particularly excellent characteristics. The developed film microrobot is extremely light (with a density of only $45 \mu \mathrm{g} / \mathrm{mm}^{2}$ ), and it can be processed easily. The film robot can be driven by a low voltage of $5 \mathrm{~V}$ using PWM control. Our experiments indicated that it is possible to perform precise position control repeatedly with a relative standard deviation of $4.7 \%$ in the worst case. In particular, the film robot showed stable repetitive actuation performance (greater than 8000 actuations). To demonstrate the potential and simplicity of this process, we fabricated two-joint, multi-finger, and gripper robots with multiple degrees of freedom and a mobile illuminator film robot. Each could be precisely manipulated with 
multiple degrees of freedom, thus indicating that the proposed process was simple and could easily be used to form a variety of robots through patterning. Additionally, both the OPP and PI sides of the film can be used to form electrodes by sputtering or other conventional lithography techniques. Electronic circuits or sensors can be then integrated to these films. The films could be used as a kinetic flexible printed circuit for various electronic circuits, computers, and probe chips for biological and chemical experiments; these applications can be termed as "Kinetic electronics".

\section{Materials and Methods}

Fabrication of bimorph flexible actuator films. For the actuation layer of the film microrobot, we prepared a bimorph flexible actuator film by lamination bonding with four base films having different thermal and mechanical properties. Oriented polypropylene (OPP) (SO25-1, Hattori, Japan), polyimide (PI) (Kapton 50H, Dupont, USA), copy paper (WC901PET, APPJ, Indonesia), and aluminum foil (Nippaku foil, Mitsubishi-aluminum, Japan) were employed as the base films to fabricate five types of actuator films (PI/OPP, Paper/OPP, Al/OPP, Paper/PI, AI/PI). The properties related to the bimorph actuator of the base film are listed in Table 1. To fabricate the actuator film, each base film was first cut to $100 \mathrm{~mm}$ squares and rubbed on both sides by a clean room-grade paper wipe (Bemcot PS2, Asahi-kasei, Japan) with $99.9 \%$ ethanol. Then, the bonding side of the base film was washed with flowing ethanol and dried completely by placing it in pure atmosphere for $10 \mathrm{~min}$. Next, a commercial adhesive for difficult-to-adhere polymers (SU05141, KONISHI, Japan) $10 \%$ diluted with acetone was applied to the bonding side of the film. The second film was carefully overlapped from the edges of the first film to form a pair of films. A spatula was used on one side of the film to squeeze the excess adhesive and air bubbles between the films. Then, the pair of films were sandwiched between flat glass plates weighing $3 \mathrm{~kg}$. The pair of films were then placed on a flat hot plate set at the bonding temperature $\left(25^{\circ} \mathrm{C}\right.$ for all experiments described in this paper) for $2 \mathrm{~h}$. The fabricated actuator film flattened at the bonding temperature.

Thermal deformation of bimorph actuator films. For each combination of bimorph actuator films, specimens of size $40 \mathrm{~mm} \times 5 \mathrm{~mm}$ were cut to establish the relationship between curvature and temperature. The specimens were placed on a hot plate, and a video camera (C980GR, Logitech, Swiss land) was set vertically at a top-view angle to observe the deformation of each film from $20^{\circ} \mathrm{C}$ to $100^{\circ} \mathrm{C}$. The curvatures were calculated from the captured photographs.

Driving system for microrobot. The developed film microrobot was driven by a microcontroller (Arduino nano, Gravitech, USA) via a custom-made solid-state relay circuit. To eliminate the variation in actuation caused by the heater and film geometry, PWM was used for robot control. 
1 PWM power signals with an amplitude of 5-10 $\mathrm{V}$ were used for each robot finger driven with a 2 tuned duty ratio. In this experiment, a desktop DC stabilization power supply (AD-8724D, AND,

3 China) and a DC power source on a breadboard were used as power sources. All robot driving 4 and manipulation experiments were conducted using this setup. The film robot was fixed on a polystyrene plate, and robot actuation was recorded using a video camera. All experiments were conducted at approximately $20^{\circ} \mathrm{C}$ in the laboratory.

Generated force and robot power measurements. An electric balance (TW323N, SHIMADZU, Japan) was used to measure the force and power generated by the robot. The electric balance was set to zero after a plastic post was placed on its weighing dish. The accuracy of the electric balance was $\pm 0.001 \mathrm{~g}$. A downward lateral force was applied to the post by bending actuation of the robot finger. Force measurements began when at the vertical position that was just in contact with the robot finger to the post without a load.

\section{References}

1. Kim J, Campbell AS, de Avila BE, Wang J. Wearable biosensors for healthcare monitoring.

2. Sempionatto JR, et al. Eyeglasses based wireless electrolyte and metabolite sensor

3. Suzuki K, Mito G, Kawamoto H, Hasegawa Y, Sankai Y. Intention-based walking support

4. Liu Y, Pharr M, Salvatore GA. Lab-on-Skin: A Review of Flexible and Stretchable

5. Lacour SP, Wagner S, Huang Z, Suo Z. Stretchable gold conductors on elastomeric substrates. Applied Physics Letters 82, 2404-2406 (2003).

6. $\mathrm{Ng} \mathrm{TN}$, Wong WS, Chabinyc ML, Sambandan S, Street RA. Flexible image sensor array with bulk heterojunction organic photodiode. Applied Physics Letters 92, 213303 (2008).

7. Forrest SR. The path to ubiquitous and low-cost organic electronic appliances on plastic. Nature 428, 911-918 (2004).

8. Sassa F, Biswas GC, Suzuki H. Microfabricated electrochemical sensing devices. Lab Chip 20, 1358-1389 (2020).

9. Bandodkar AJ, Jia W, Yardimci C, Wang X, Ramirez J, Wang J. Tattoo-based noninvasive glucose monitoring: a proof-of-concept study. Anal Chem 87, 394-398 (2015).

10. Liu X, Lillehoj PB. Embroidered electrochemical sensors for biomolecular detection. Lab Chip 16, 2093-2098 (2016). 
11. Liao Y-T, Yao H, Lingley A, Parviz B, Otis BP. A 3-\$¥mu¥nbox\{W\}\$CMOS Glucose Sensor for Wireless Contact-Lens Tear Glucose Monitoring. IEEE Journal of Solid-State Circuits 47, 335-344 (2012).

12. Rus D, Tolley MT. Design, fabrication and control of soft robots. Nature 521, 467-475 (2015).

13. Hines L, Petersen K, Lum GZ, Sitti M. Soft Actuators for Small-Scale Robotics. Adv Mater

14. Bar-Cohen Y, Anderson IA. Electroactive polymer (EAP) actuators-background review.

15. Dubois $\mathrm{P}$, et al. Microactuators based on ion implanted dielectric electroactive polymer

16. Konishi S, Kawai F, Cusin P. Thin flexible end-effector using pneumatic balloon actuator.

17. Shepherd RF, et al. Multigait soft robot. Proc Natl Acad Sci U S A 108, 20400-20403

18. Wehner $M$, et al. An integrated design and fabrication strategy for entirely soft, autonomous robots. Nature 536, 451-455 (2016).

19. Li Q, Liu C, Lin YH, Liu L, Jiang K, Fan S. Large-strain, multiform movements from designable electrothermal actuators based on large highly anisotropic carbon nanotube sheets. ACS Nano 9, 409-418 (2015).

20. Potekhina A, Wang CH. Review of Electrothermal Actuators and Applications. Actuators 8, 69 (2019).

21. Yao S, Cui J, Cui Z, Zhu Y. Soft electrothermal actuators using silver nanowire heaters. Nanoscale 9, 3797-3805 (2017).

22. Liu X, Liu J, Lin S, Zhao X. Hydrogel machines. Materials Today 36, 102-124 (2020).

23. Yoshida $\mathrm{R}$, et al. Comb-type grafted hydrogels with rapid deswelling response to temperature changes. Nature 374, 240-242 (1995).

24. Martinez RV, Glavan AC, Keplinger C, Oyetibo AI, Whitesides GM. Soft Actuators and Robots that Are Resistant to Mechanical Damage. Advanced Functional Materials 24, 3003-3010 (2014).

25. Sassa F, Hayashi K. Flexible Thermal Actuator Film for Monolithic Soft Micro Robot Process. In: IEEE SENSORS 2018). IEEE (2018).

26. Zolfagharian A, Kouzani AZ, Khoo SY, Moghadam AAA, Gibson I, Kaynak A. Evolution of 3D printed soft actuators. Sensors and Actuators A: Physical 250, 258-272 (2016).

27. Schmitt F, Piccin O, Barbe L, Bayle B. Soft Robots Manufacturing: A Review. Front Robot A/ 5, 84 (2018). 
28. Timoshenko S. Analysis of bi-metal thermostats. J Opt Soc Am Rev Sci 11, 233-255 (1925).

29. Suhir E. Interfacial Stresses in Bimetal Thermostats. Journal of Applied Mechanics 56, 595-600 (1989).

30. Croxatto A, Prod'hom G, Faverjon F, Rochais Y, Greub G. Laboratory automation in clinical bacteriology: what system to choose? Clin Microbiol Infect 22, 217-235 (2016).

31. Arakawa $\mathrm{T}$, et al. Mouthguard biosensor with telemetry system for monitoring of saliva glucose: A novel cavitas sensor. Biosens Bioelectron 84, 106-111 (2016).

32. Ciui B, et al. Cavitas electrochemical sensor toward detection of $\mathrm{N}$-epsilon (carboxymethyl)lysine in oral cavity. Sensors and Actuators B: Chemical 281, 399-407 (2019).

33. McKeen LW. Film Properties of Plastics and Elastomers, 4th Edition (2017).

34. Karger-Kocsis J. Polypropylene An A-Z reference (1999).

\section{Acknowledgement}

This work has been financially supported by JSPS KAKENHI Grant Number 19H05680, and Adaptable and Seamless Technology transfer Program through Target-driven R\&D (A-STEP) from Japan Science and Technology Agency (JST) Grant Number JPMJTM20GW, and MEXT Initiative for Realizing Diversity in the Research Environment.

\section{Author Contributions}

F.S. designed research; S.Z. and F.S. performed research; S.Z., J.W. and F.S. contributed new reagents/analytic tools; S.Z., J.W., K.H. and F.S. analyzed data; S.Z., J.W. and F.S. wrote the paper.

\section{Competing interests}

The authors declare no competing interests.

\section{Additional information}

Supplementary information: See the supplementary material for the actuation of developed film microrobots.

Correspondence and requests for materials should be addressed to J.W and F.S.

\section{Data Availability}

The data that support the findings of this study are available from the corresponding author upon reasonable request. 


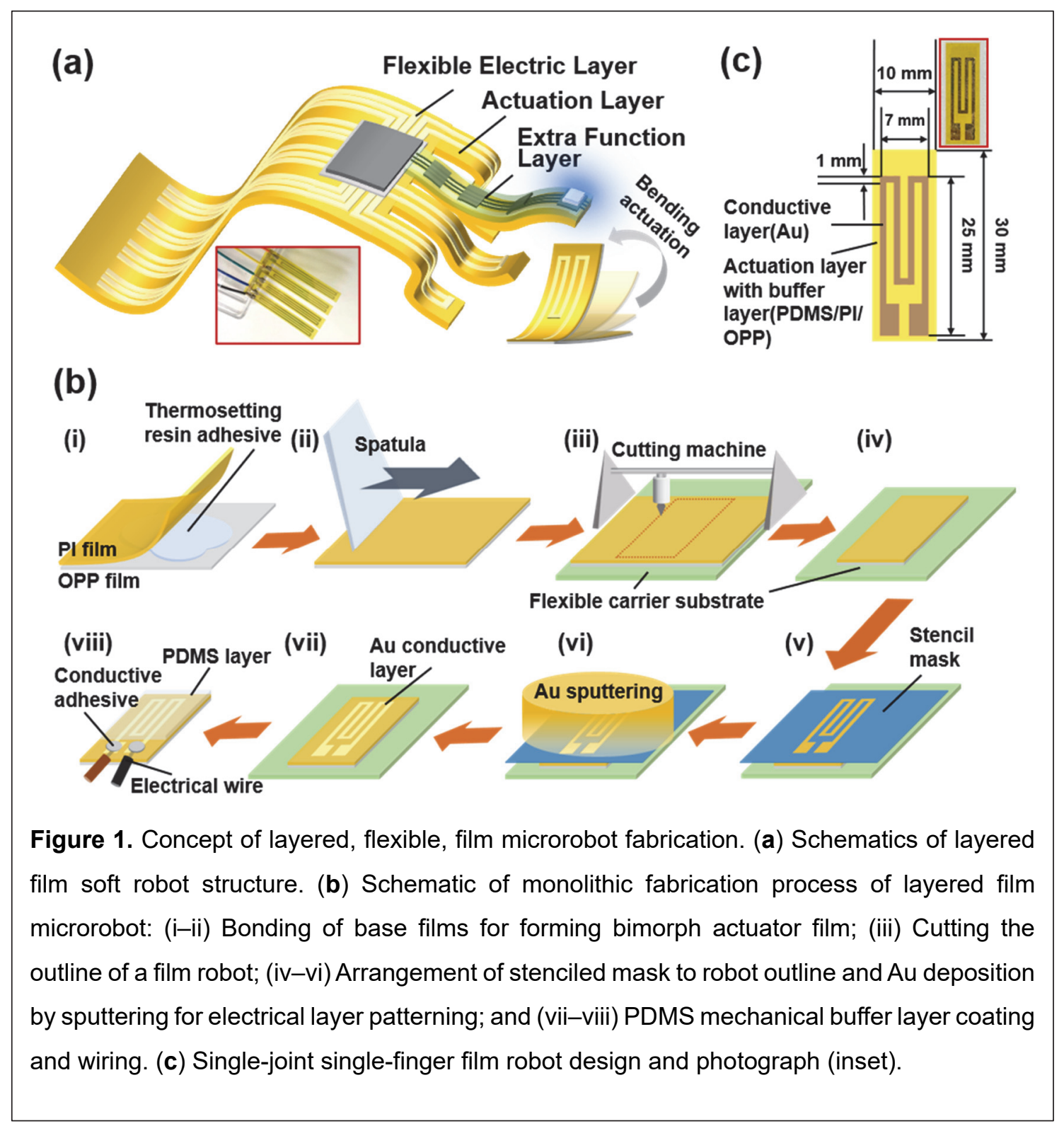


(a)

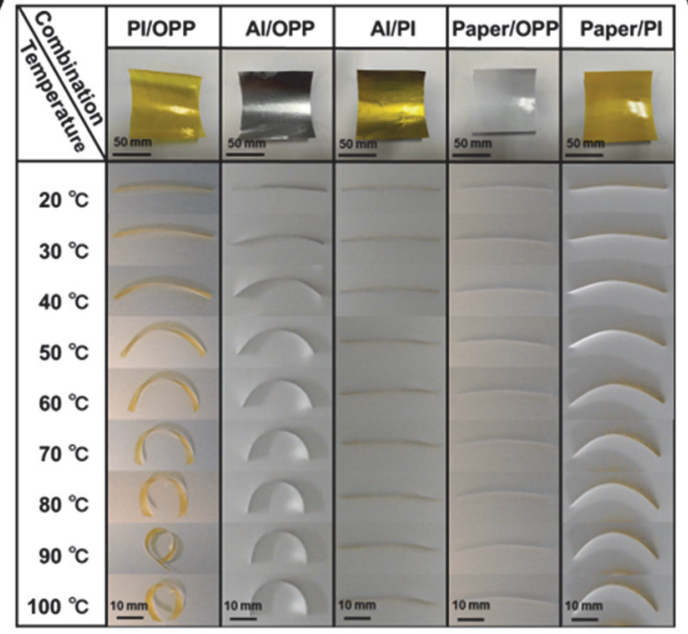

(b)

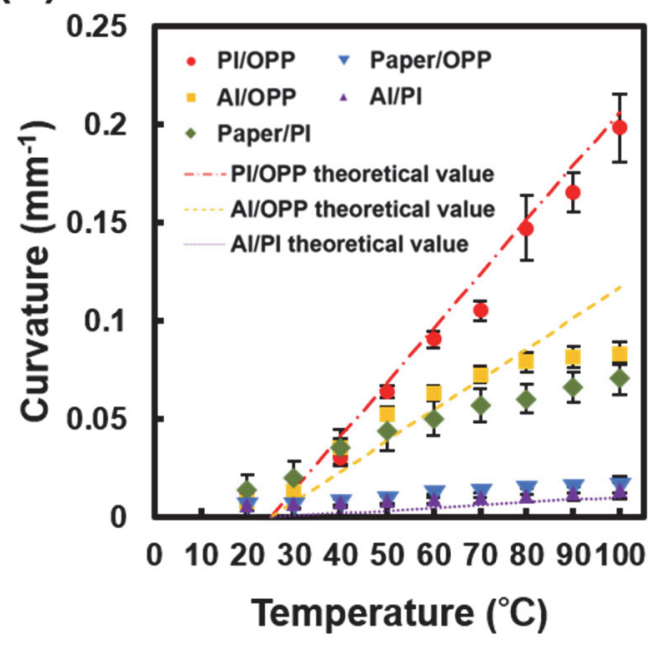

Figure 2. Evaluation of thermal deformation of bimorph actuator films. (a) Photographs of fabricated bimorph actuator films and their deformations. (b) Relationship between curvature and temperature. Bars represent standard deviation $(n=5)$. 
(a) Duty $0 \%$ Duty $20 \% \quad$ Duty $\mathbf{4 0 \%}$ Duty $60 \%$ Duty $80 \% \quad$ Duty $100 \%$
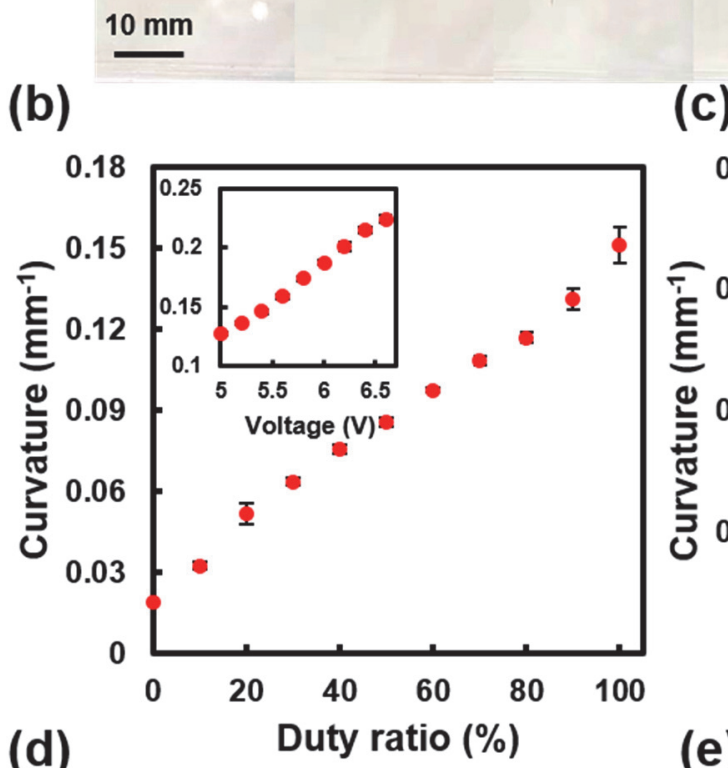

(c)
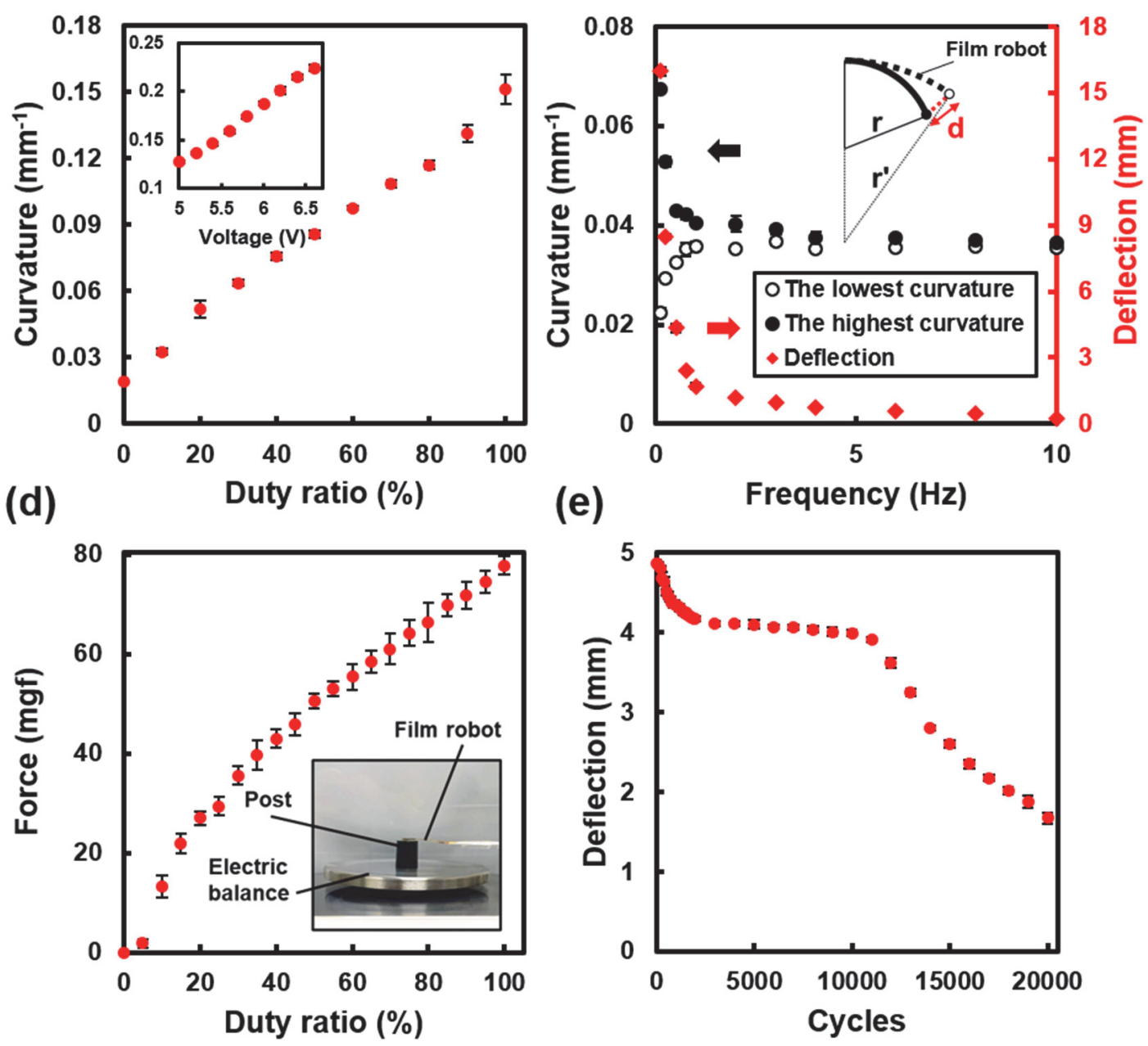

Figure 3. Actuation characteristics with single-finger film microrobot. (a) Photograph of robot actuation with different duty ratios with a $5 \mathrm{~V}$ PWM power supply. (b) Dependency of curvature on supplied power (5 V PWM) and on DC voltage change at $100 \%$ duty ratio (inset). (c) Frequency response of actuator deformation shown as minimum and maximum curvature on the amplitude (left axis) and tip deflection (right axis). (d) Generated force at zero deflection point. and photograph of measurement setup (inset). (e) Repetitive actuation durability of the robot on 20000 bending motions. Bars represent standard deviation $(n=5)$. 


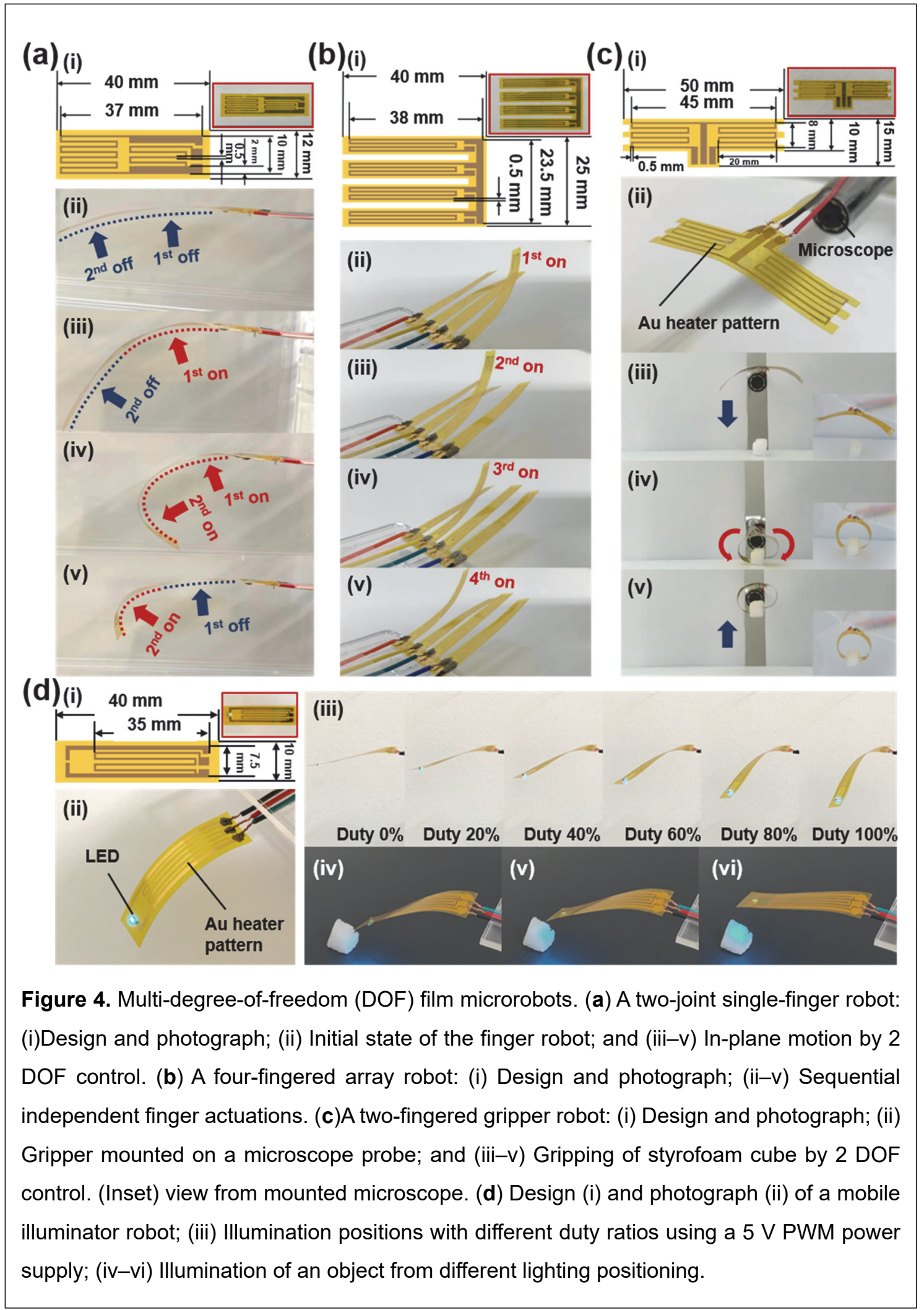


1 Table 1. Thermal and mechanical properties of the film material for bimorph actuator films.

\begin{tabular}{|c|c|c|c|}
\hline Material & $\begin{array}{l}\text { Thickness } \\
(\mu \mathrm{m})\end{array}$ & $\begin{array}{l}\text { Young's } \\
\text { (GPa) }\end{array}$ & $\begin{array}{c}\text { Coefficient of thermal } \\
\text { expansion }\left(1 /{ }^{\circ} \mathrm{C}\right)\end{array}$ \\
\hline $\mathrm{Pl}^{33}$ & 12.5 & 3.5 & $2.0 \times 10^{-5}$ \\
\hline OPP 34 & 12 & 1.9 & $8.0 \times 10^{-5}$ \\
\hline $\mathrm{Al}$ & 20 & 69 & $2.4 \times 10^{-5}$ \\
\hline Paper & 90 & N.A. & N.A. \\
\hline
\end{tabular}

2 


\section{Supplementary Files}

This is a list of supplementary files associated with this preprint. Click to download.

- S1Singlefingeredfilmrobot.mp4

- S2Twojointsinglefingeredfilmrobot.mp4

- S3Fourfingeredarrayrobot.mp4

- S4Twojointgripperrobot.mp4

- S5Movingilluminatorfilmrobot.mp4

- NatureComSassaSI.pdf 